\title{
ZUR HEIMA'T DER VOLCAF.
}

Müllenhoff hat bekanntlich im zweiten teile der Deutschen altertumskunde aus den schon in M. WT. Dunckers Origines Germanicae vollständig gesammelten berichten über die wohnsitze der Volcae zu erweisen versucht, dass die heimat dieses volkes bis gegen das 4. jh. 'an der Weser abwärts' und dann im Maintale gelegen habe $(2,279)$. Diese ansicht ist von Much als unrichtig erwiesen, aber noch verfehlter scheint mir seine an ihrer statt aufgestellte hypothese, dass Mähren die heimat der Volcae gewesen sei.

An den zwei ersten Keltenzügen hatten die Volcae keinen anteil; erst der dritte, der galatische, führte sie in den horizont der Griechen und Römer hinein. Nach dessen ablauf finden wir das volk zersplittert in drei oder vier weit auseinander liegenden gegenden wohnhaft. Erstens in Narbonensis zwischen Rhône und Pyrenäen, wo sie zuerst bei Hannibals Alpenzuge erwähnt werden, aber schon anfangs des 3. jh.'s angekommen sein müssen (Duncker s. 33). Dann in Kleinasien, wo ein teil des völkerschwarmes, der seit 281 die thrakischgriechische halbinsel durchzogen hatte, nene wohnsitze gefunden hatte. Und drittens an den Ost-Alpen und (oder) an der Hercynia silva.

Justin berichtet $(32,3)$ : Namque Galli bello adversus 1)clphos infeliciter gesto, ... pars in Asiam pars in Thraciam c.xtorres fugerant. Inde per eadem vestigia qua venerant antiguam putriam repetivere. Ex his manus quaedam in conflucnte I)anubii et Savi consedit Scordiscosque se appellari voluit. Tirtosages autem, cum in antiquam patriam Tolosam renissent.... Exx gente Tectosagorum non mediocris populus macdac dulcedine Illyricum repetivit spoliatistuc Istris in Pannonia consedit 
I)er letzie teil des berichtes steht vereinzelt da: kein anderer antor weiss etwas von 'lectosages in Pannonien. Aber die 110ti\% wird dadurch noch nicht verwerflich. Sowol der geschichtsschreibung wie der sage galt das tolosanische als die heimat des ganzen volkes der Volcae, und zugleich leitete die erstere das tolosanische gold vom Bremnuszuge nach Delphi her. Iaraus erklärt sich die fassung des berichts Justins, dass der in Pannonien angesiedelte teil erst von Narbonensis nach Griechenland, dann zurïck nach 'I'olosa und dann wider nach Illyrien gezogen sei. Nach ausmerzung dieses augenscheinlichen von der tradition betreffend Tolosa veranlassten irrtumes bleibt die an sich nicht unwahrscheinliche nachricht übrig, dass die Tectosages ausser in Narbonensis und in Galatien, auch zum teil in Pannonien in der nähe der Istri, d. h. im obern Sautale, sich niedergelassen hatten.

Wir finden aber das volk bei Caesar noch an einer vierten stelle, nämlich an der Hercynia silva, ansässig. Dieser sagt (BG. 6, 24): Ac fuit antea tempus, cum Germanos Galli virtute superarent, ultro bella inferrent, propter hominum multitudinem agrique inopiam trans Rhenum colonias mitterent. Itaque ea quae fertilissima Germaniae sunt loca circa Hercyniam silvam, quam Eratostheni et quibusdam Graecis fama notam esse video, quam illi Orcyniam appellant, Volcae Tectosages occupaverunt atque ibi consederunt; quae gens ad hoc tempus his sedibus sese continet summamque habet iustitiae et bellicae laudis opinionem. Schon Ben. Niese hat diese nachricht mit der Justins verbunden (Zs.fda.42,142), aber nur um beide gänzlich zu verwerfen. Nach ihm soll durch die Tectosages Caesars und Justins 'ursprünglich wol nur die herkunft der pannonischen Kelten erklärt werden', und 'diese Volcae am hercynischen walde ganz und gar der fabel zuzuweisen sein'. Es ist aber schwerlich einzusehen, wie 'die wanderungssage' dazu kommen konnte, in Pannonien die existenz eines zweiges der Volcae T'ectosages zu fingieren, mit dem zwecke, die herkunft anderer pannonischer Keltenstämme zu erklären.

Müllenhoff meinte mit recht, Caesar habe, der sage und der tradition folgend, die Tectosages an der Hercynia für eine colonie ihrer stammesgenossen an der Cevenna gehalten (2,276), und habe die sage vom Sigovesuszuge im sinne gehabt (204), 
wonach zur gleichen zeit, als die Gallier unter Bellovesus iiber die Alpen in Italien eindrangen, ein anderer teil desselben heeres sich unter Sigovesus ostwärts gewant habe und in die hercynischen wälder ausgezogen sei (261). Aber unberechtigt ist der daraus von Müllenhoff gezogene schluss, dass alles an dem berichte verwerflich sei ausser dem wohnsitze eines teiles der Tectosages an der Hercynia. Beseitigt man Caesars oratorische fassung des berichts, so besagt der kern nichts anderes, als dass die Tectosages noch zu seiner zeit an der Hercynia wohnten, aber da keine autochthonen waren, sondern aus dem alten Keltenlande stammten. Warum diese offenbar auf gallischer tradition beruhende notiz fabelhaft sein soll, ist nicht einzusehen. Im gegenteil stimmt sie $\mathrm{zu}$ allem, was wir übrigens von den wohnsitzen des volkes wissen und zu den resultaten der untersuchungen über die Keltenzüge (Duncker, Müllenhoff, Hirschfeld, Niese u.s.w.).

Nach den Volcae an der Hercynia ist überall herumgesucht worden: den meisten anklang fanden noch Müllenhoffs hypothese, sie hätten im Maintale gewohnt, und die Muchs, ihre heimat sei Mähren. Beide hielten jene gegenden für die, woraus die Volcae zu anfang des 3.jh.'s einerseits nach Narbonensis, andererseits nach der thrakisch-griechischen halbinsel abgezogen seien. Dem widersprechen aber Caesars worte quae gens ad hoc tempus his sedibus sese continet, denn die Volcae von Narbonensis und Galatien können unmöglich aus einem ver sacrum hervorgegangen sein und nur aus dem auszuge des ganzen volkes aus seiner vorigen heimat erklärt werden. Der fehler liegt m. a. n. hierin, dass man bis jetzt nicht erwogen hat, was in Caesars notiz unter Hercynia silva zu verstehen ist.

Die stelle Caesars lag Tacitus vor bei c. 28 der Germania: Validiores olim Gallorum res fuisse summus auctor divus Julius tradit, coque credibile est etian Gallos in Germaniam trans: yressos. Worauf folgt: Igitur inter IIercyniam silvam I7hen umque et Moenum annes Helvetii, ulteriora Boii, Gallica utraque gens, tenuere. Manet adhuc Boihacmi nomen significatgue loci veterem memoriam, quamvis mutatis cultoribus. An diestr stelle ist Hercynia silva natürlich die Rauhe Alb, nicht. wir. sonst bei Tacitus (Germ. 30. Ann. 2, 40) und den anderen nuch- 
rassarischen autoren, das dentsche mittelgebirge, was andeutet, lass 'Tacitus die stelle einem ältern autor entnommen, der die rebirge nïrdlich vom Main noch nicht kannte. Ich vermute, latss seine quelle eben dieselbe stelle Posidonius war, woraus

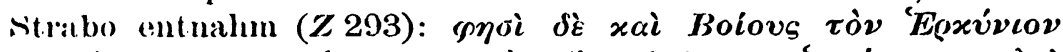

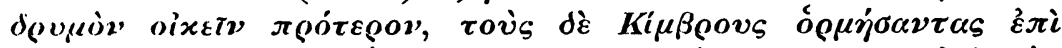

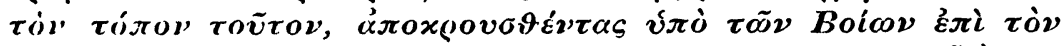

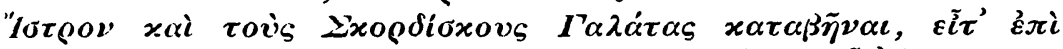

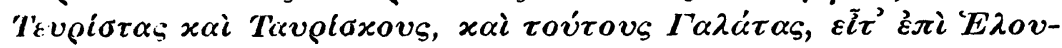
nтriovs. I)araus geht nur hervor, dass Posidonius die gebirge Böhmens Hercynia nannte, aber nicht ob er die Rauhe Alb oder die gebirge nördlich vom Main unter dem namen mitbegriff, und in anbetracht seines alters ist das erstere wahrscheinlicher. Jedenfalls aber hatte Tacitus einen autor vor sich, der ihn veranlasste die ihm ebenfalls vorliegende stelle Caesars, welche als keltisches volk an der Hercynia nur die Volcae Tectosages nannte, zu verwerfen, denn sonst hätte er hier ohne zweifel auch diese miterwähnt.

Caesars notiz stammt augenscheinlich aus einer griechischen quelle, aber nicht aus Posidonius, weil sonst Strabo die Tectosages wol auch genannt haben würde. Erwägt man aber, dass die angeführte stelle des Posidonius die älteste uns bekannte ist, worin der name 'Eoxv́vıc von den Alpen') auf die Rauhe Alb und die nordöstlich anschliessenden gebirge übertragen war (DA. 1,432), und dass Caesar in seiner vorlage noch die

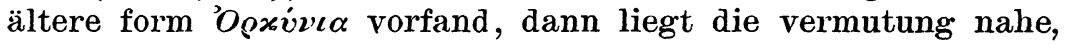
dass diese unter dem namen noch die Alpen verstand, was Caesar natürlich entgehen musste. Und hält man nun die stelle, so gedeutet, neben der von Justin aus Trogus ausgezogenen, so zeigt sich sofort, dass beide aus derselben quelle

1) Es scheint mir nicht überflüssig hervorzuheben, dass die anwendung des keltischen namens auf die Alpen natürlich nur von der geographischen unkenntnis der älteren Griechen herrührt. Im keltischen muss Perkunia von anfang an das deutsche mittelgebirge zwischen Rhein und Weichsel bezeichnet haben; denn nur so lässt sich die entlehnung von fairguni im gotischen mit der bedeutung des namens bei den nachcaesarischen aútoren vereinigen. Jedenfalls ist es unrichtig, den namen auf das Erzgebirge oder die böhmischen randgebirge zu beschränken, und ist mithin auch der schluss hinfällig, dass die Germanen den namen nur an der grenze Böhmens oder Mährens aus dem keltischen entlehnen konnten. 
geflossen sein müssen, welche etwa dieses enthielt, dass die Tectosages sich von Gallien nach der Orcynia gewant und sich dort nach besiegung der Istri niedergelassen hatten. ${ }^{1}$ ) Caesar hat dann die Istri weggelassen, weil er wusste, dass diese nicht an (seiner) Hercynia wohnten, und überdies für den zweck seines excerptes keinen wert hatten, während Trogus umgekehrt die Orcynia ausmerzte, weil er wusste, dass bei den Istri nur von den Alpen, nicht von (seiner) Hercynia die rede sein komnte.

Ob Caesars worte quae gens ad hoc tempus his sedibus sese continet von ihm selbst herrühren oder aus der gemeinsamen quelle stammen, lässt sich schwerlich entscheiden. Es wäre möglich, dass das volk schon vor Caesar verschwunden war, und z. b. das los der Boier, der vernichtung durch die Daker Boirebistas (Strabo 1213 ), geteilt hatte, aber ebenso möglich und m. a. n. wahrscheinlicher ist, dass sie bei Ptolemaeus widerkehren unter dem erst bei diesem vorkommenden namen Latovici. Jedenfalls aber ist das fehlen der 'lectosages bei Strabo und Ptolemaeus kein grund, wie Niese will, um die notizen Caesars und Justins ganz der fabel zuzuweisen.

Aus der gemeinsamen quelle Caesars und Justins muss auch die enge verbindung stammen, worin bei letzterm der zug der Tectosages nach Illyrien zum Galaterzuge nach Delphi stand, wobei es gleichgiltig ist, von wem die augenscheinliche umkehrung der zeitfolge der zwei züge herrührt. Die verbindung aber bestätigt die vermutung Dunckers (28), dass die grosse Keltenbewegung an den Alpen um 300, woran die erinnerung bei Livius $(10,10)$ und Polybius $(2,19)$ erhalten blieb, den stoss gab zum Galaterzuge. Ist die vermutung richtig, dann gehörte zum Gallorum ingens exercitus, der nach Livius in 299 in Etrurien eingefallen war, auch der non mediocris populus ex gente Tectosagorum, der nach. Justin nachher die Istri besiegte und sich in Illyrien niederliess. Von ihren neuen dortigen wohnsitzen muss dann in 281 der vorstoss zum Galaterzuge ausgegangen sein, denn die Volcae bildeten ommimm.

1) Diese urspriingliche quelle muss also ä]ter als Posidonius gewesen sein, aber das hindert nicht die ansicht Gutschmids, dass die hauptquelle Justins Timagenes gewesen sei, noch die Nieses, dass Nepos die grmeinschaftliche nïchste quclle fiir diesen passus Justins und Livins" whr. 
consensu den schwerpunkt des zuges, und das zurückbleiben cines teils des stammes in Illyrien steht der annahme gar nicht entgegen. lis ist begreiflich, dass die bei Callimachus und l'ausinias erhaltene sage (1)A.2, 272) die zwei kurz aufcinamler folgenden rüge desselben volkes miteinander verband und es aus lem äussersten westen Europas nach Griechenland kommen liess. Und zugleich wird dadurch die scharfsinnige vermutung I)unckers (33) bestätigt, die Volcae an der Cevenna seien da ums jahr 300 angekommen. Livius erzählt, dass die Gallier mit der bestimmten absicht gekommen waren, neue wohnsitze zu erlangen, ut tandem aliqua sede certa consistant. Es ist mithin wahrscheinlich, dass die Volcae sich erst, nachden die Etrusker sich ihren abzug erkauft hatten, in zwei züge geteilt haben, wovon der eine sich ostwärts nach Mlyrien und der andere westwärts nach Narbonensis wandte, und es scheint mir selbst nicht unmöglich, dass das zwei jahrhunderte später von Caepio geraubte aırum tolosanum grösstenteils aus der ingens pecunia stammte, welche die Galli (Volcae) in 299 aus Etrurien über die Alpen mitgenommen hatten.

Den wohnsitzen der Volcae im Maintale und in Böhmen bei Müllenhoff und in Mähren bei Much ist hiermit glaube ich der boden geschwunden. Nach Griechenland kam das volk aus Pannonien, und nach Pannonien und Narbonensis aus Etrurien. Woher aber kam es, als es im jahre 299 in Etrurien einfiel? Hier müssen wir anknüpfen an Müllenhoffs schöne und folgenreiche entdeckung (2, 279): 'dass die Volcae einst die unmittelbaren nachbarn der Germanen waren und aus deren nächster nähe abgerückt sind, unterliegt mindestens keinem zweifel, weil die Germanen nach ihnen den ganzen keltischen volksstamm benannt haben'. Dabei muss man aber im auge behalten, dass der name $W a l_{\chi} \bar{\partial} z=$ 'Kelten' sich nu' im westgermanischen findet, während er im ostgermanischen fehlt. $\left.{ }^{1}\right)$ Die Volcae waren also wahrscheinlich die ursprünglichen keltischen nachbarn der Westgermanen.

1) Dass Wlahu im slavischen aus dem deutschen entlehnt ist, beweist noch nicht, wie Müllenhoff will, dass die Slaven den namen von den Goten oder Ostgermanen entlehnt haben müssen. Die entlehnung kann ebensogut geschehen sein, nachdem die Slaven nach dem abzuge der Goten und anderen Ostgermanen zu nachbarn der Westgermanen geworden waren, und 
An den zwei ersten Keltenzügen hatten die Volcae keinen anteil gehabt. Der erste, nach Müllenhoff im $6 . j \mathrm{~h}$. anzusetzen, fïgte dem alten Keltenlande das vorher nur von Iberiern und Liguriern bewolnte gebiet zwischen Loire und Garonne hinzu, und führte den stamm dann nach Iberien, wo er den Griechen zuerst bekaunt wurde unter dem namen Celtae.1) Er gieng also vermutlich von der Loire aus. Der zweite zug gieng zu anfang des 4.jh.'s über die Alpen nach Italien, und mag er nun nach der bei Livius erhaltenen tradition ebenfalls von dem mittleren Gallien, oder nach der modernen ansicht Bertrands, d'Arbois de Jubainvilles und Nieses (a.a.o.s. 151) von den Donauländern ausgegangen sein, jedenfalls wird die bei Justin erhaltene tradition, der zug sei aus einem ver sacrum hervorgegangen, bestätigt durch den umstand, dass sich damals im cisalpinischen Gallien eine anzahl völker niederliess, wovon mehrere namensvetter im transalpinischen zurückliessen. Für sich allein berechtigen die zwei züge nur zu dem schlusse, dass die ursprünglichen grenzen zwischen Germanen und Kelten gegen den schluss des 5. jh.'s noch nicht verschoben waren. ${ }^{2}$ )

Ein jahrhundert später aber kommen die ursprünglichen keltischen nachbarn der Westgermanen nach Italien, ut tandem aliqua sede certa consistant. Sie sind aus ihrer urheimat vertrieben, ohne zweifel durch die Germanen, denn nach dem jahre 300 waren diese nie und nirgends ihre nachbarn, so dass die entlehnung ihres namens nur vorher stattgefunden liaben kann, und ihre vertreibung gegen den schluss des 4. jh.'s geschehen sein muss. Treffend stimmt hierzu das resultat der untersuchungen Kossinnas (Ueber den ursprung

es wäre höchst auffallend, dass sich keine einzige spur des namens in den ostgermanischen sprachresten erhalten hat, wenn er da wirklich existiert hätte.

1) In meiner abhandlung De civitates van Gallië, in den Verhh. der k. akad. v. wetensch., afd. letterk., n. r. 2, no. 1 (Amst. 1898, s. 48) hahe ich gezeigt, dass der zweifel Kieperts und Müllenhoffs an der wahrheit der äusserung Caesars, dass der keltische stamm des mittleren (Galliens ipsorum lingua Cellae appellantur; unberechtigt, ist.

2) Obiges beanstandet, nicht, die auch $\mathrm{m}$. a. n. richtige bypothere, dass die küsten zwischen Weser und Rhein schon damals von Ingrvionen lo.siedclt waren. 
5.14 MITTIRR, ZITR IIEIMAT IDER VOICAE. - HORN, ZU BEITR. 24, 403.

des Germanennamens, Beitr. 20, 297, und Die vorgeschichtliche ausbreitung der Germanen in Deutschland in der Zs. d. ver. f. volksk. 1896, s.9), dass das gebiet \%wischen Leine und Rhein seit etwa 300 steine germanische bevölkerung erhalten hat, und es scheint mir deshalb unmöglich, die urheimat der Volcae vor ihrem aufbruch nach dem süden gegen 300 anderswohin zu verlegen als nach jenem gebiet, woraus sie durch die Istaevonen vertrieben wurden.1) An der Weser, Aller und Leine lag jahrhunderte hindurch die grenze zwischen Kelten und

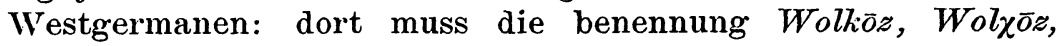
$\left.W a l_{\chi} \bar{z} z^{2}\right)=$ 'Kelten' entstanden sein.

ROTTERDAM, november $1899 . \quad$ S. MULLER.

\section{ZU BEITR. 24, 403.}

Nachträglich finde ich, dass bereits Scherer im jahre 1869 in der besprechung von Lexers Wörterbuch (Zs. f. österr. gymn. $20,831 \mathrm{f}$. = Kleine schriften 1,379) alde mit ahd. erdo zusammengestellt hat. $\mathrm{Er}$ sagt: 'Die zurückführung von alde, alder (nebenform von ocler) auf al »ander " halte ich nicht für glücklich, das dld der hochdeutschen grundform eddô ist singulär genug, um singuläre lautvertretungen begreiflich $\mathrm{zu}$ machen. Man mag hier zunächst an althochdeutsches erdo und das vereinzelte $l$ für $r$ denken.'

DARMSTAD'T, 1. august 1899.

WILHELM HORN.

1) Wenn die durch Timagenes bei Ammian $(15,9,4)$ erhaltene druidensage, dass die Kelten zum teil ab insulis cxtimis et tractibus transrhepanis stammten, jenem in Narbonensis erzählt war, so könnte darin eine volkssage der Volcae stecken.

2) Für die zeitbestimmung der lautverschiebung ist das gewonnene datum unerheblich, da die umbildung von Wolkzz in Walxoz ebensogut vor als nach dem abzuge der Volcae stattgefunden haben kann. 